\title{
CAN GUT MICROBES PLAY A ROLE IN MENTAL DISORDERS AND THEIR TREATMENT?
}

\author{
Klara Latalova, Miroslav Hajda \& Jan Prasko \\ Faculty of Medicine and Dentistry, University Palacky Olomouc, Olomouc, Czech Republic \\ Department of Psychiatry, University Hospital Olomouc, Olomouc, Czech Republic
}

received: 22.11.2015;

revised: 3.11.2016;

accepted: 29.12.2016

\begin{abstract}
SUMMARY
The gut microbes, collectively called microbiota, are linked to the brain through a bidirectional system that involves the vagus nerve, the immune system, and various neurotransmitters. Stress response, memory functions, social behavior, and mood are modulated by microbiota. Furthermore, microbiota play a role in the development of the central nervous system. These features, established largely in rodent studies, have informed hypotheses about the role of microbiota in human psychiatric disorders. Microbiota affect phenomena that are known to be parts of the depression phenotype, such as exaggerated response to stress and inflammatory features. Furthermore, the role of microbiota in neurodevelopment and in the modulation of social behavior suggests the possibility of its role in autism spectrum disorder and in schizophrenia. If altered, microbiota play a role in psychiatric disorders, then efforts to normalize the gut microbial population by the ingestion of probiotics (live bacteria) could have antidepresssant or antipsychotic effects. Testing such hypotheses in translational human studies is a matter of future research.
\end{abstract}

Key words: microbiota - gut microbiome - brain - depression

\section{Introduction}

"Can the bacteria in your gut explain your mood?" Ten years ago, that question would have sounded like a joke. Not anymore. The quoted question is in fact the title of a full-scale review by P.A. Smith, published in The New York Times on June 23, 2015. The subtitle: "The rich array of microbiota in our intestines can tell us more than you might think." We agree.

It has become clear that the gut microbes, collectively called the gut microbiota, (and their genes, collectively called the gut microbiome) exert effects on the brain and vice versa. Some of the effects of microbiota can be emulated by the ingestion of probiotics (live bacteria, some of which may have health benefits). Effects of microbiota on behavior of rodents have been extensively examined, and preliminary studies in humans have emerged. The US National Institute of Mental Health is inviting grant applications to study basic mechanisms of gut-brain interactions.

In summary, this line of research has captured the interest of the scientific community and the public in the US and elsewhere. As far as we know, nothing has been published about this topic in the Czech scientific literature. Here we provide a mini-review.

\section{The gut microbiota}

Fetal gut is sterile. Its initial colonization during and after birth is sourced by the maternal microbiota. The microbiota in the adult human gut consist of approximately $1 \mathrm{~kg}$ of bacteria, viruses, protozoa, fungi, and archaea. Microbiota's essential functions include food digestion, fighting pathogens, and synthesis of vitamins.
There is a complex bidirectional communications network between the gut and the central nervous system, some of which depends on the activation of the vagus nerve. The immune system transduces the effects of microbiota on the brain.

On the other hand, germ-free (GF) mice have an overactive response of the hypothalamus-pituitary-adrenal axis (HPA) to stress (Sudo et al. 2004). Moreover, GF mice also exhibited reduced brain-derived neurotrophic factor (BDNA) expression levels in the cortex and hippocampus.

The reduced BDNF expression in the cortex and the hippocampus found by Sudo et al. (2004) would be expected to yield memory impairments. Indeed, such impairments were found in GF mice (Gareau et al. 2011).

Microbiota affect the levels of neurotransmitters that modulate numerous aspects of brain activity. It influences the metabolism of tryptophan, the precursor of serotonin. Various gut microorganisms can produce dopamine, norepinephrine, gamma-aminobutyric acid (GABA), and acetylcholine (Lyte 2013). More details concerning the gut microbiota and the brain can be found elsewhere (e.g. Mayer et al. 2014, Sampson \& Mazmanian 2015).

\section{Microbiota and mental disorders}

\section{Depression}

Hypothalamus-pituitary-adrenal (HPA) axis hyperactivity in response to stress and in resting condition has been, until recently, the most extensively studied abnormality associated with major depressive episodes. It appears to be associated with melancholia, psychosis, 
and suicide risk. As mentioned above, hyperactive HPA stress response was observed in GF mice (Sudo et al. 2004). The exaggerated HPA stress response by GF mice was reversed by treatment with Bifidobacterium infantis. Importantly, the enhanced HPA response of GF mice was partly corrected by reconstitution with faeces of control mice at an early stage, but not at a later stage, which therefore indicates that exposure to microbes at an early developmental stage is required for the HPA system to become fully susceptible to inhibitory neural regulation. Thus, microbiota can affect the postnatal development of the HPA stress response in mice (Sudo et al. 2004). The response was reversed by a probiotic, suggesting a role of microbiota in the modulation of stress response.

Stress results in an alteration of gut barrier function. This may lead to the passage of various molecules, and perhaps bacteria (Sudo et al. 2004), from the gut into the bloodstream. The immune system responds by a pro-inflammatory reaction, generating high levels of pro-inflammatory interleukins IL-1 and IL-6 (Dinan et al. 2013).

It is noteworthy that individuals with major depression have significantly higher concentrations of IL-6 in comparison with controls, as demonstrated by a metaanalysis of 16 studies (Dowlati et al. 2010). In contrast, some antidepressants, including selective serotonin reuptake inhibitors, have negative immunoregulatory effects by increasing relative amounts of IL-10, a cytokine that suppresses inflammation. These results were obtained by incubating diluted whole blood from 17 normal volunteers with fluoxetine (Maes et al. 2005). Perhaps similarly, immunoregulatory effects of probiotic organisms are thought to be mediated, in part, by the synthesis of IL-10 (Dinan et al. 2013).

Thus, simplifying a very complex picture, HPA hyperactivity characterizes depression, and this hyperactivity may be mediated by a pro-inflammatory reaction to stress set off by anomalous microbiota. If so, probiotics might have antidepressant effects (Dinan \& Cryan 2013). Systematic controlled studies of probiotics for the treatment of major depression have not yet been published.

The selection of the appropriate probiotic for such treatment trial should be governed by an effort to find out what differences, if any, exist between the "depressed" microbiota and those observed in healthy controls. The probiotic tested in such trial should aim at correcting those differences. Thus, an initial approach facilitating the design of such studies could be an investigation of the microbiota in depressed patients.

At least one study of the microbiota in depression has already been done. In Finland, the compositions of microbiomes in the fecal samples of 37 depressed patients and 18 controls were examined (Naseribafrouei et al. 2014). Several types of microorganisms were significantly over- or underrepresented in the samples of depressed patients. The interpretation of these results seems difficult, particularly because of the very large variety of microorganisms present. This interesting pioneering study awaits replication in larger samples of human subjects.

Another initial approach to studies of probiotics in depression is to test their effects on mood in normal volunteers. Healthy volunteers $(\mathrm{N}=66)$ participated in a double-blind, placebo-controlled, randomized parallel group study (Messaoudi et al. 2011). A probiotic formulation containing Lactobacillus helveticus R0052 and Bifidobacterium longum R0175 was administered for 30 days. The subjects' mood was assessed with several rating scales. The probiotic significantly reduced psychological distress, somatization, depression, and angerhostility.

In another study, for a 3-week period, either a probiotic milk drink containing Lactobacillus casei Shirota or a placebo, were consumed daily by 132 healthy volunteers (Benton et al. 2007). Mood and cognition were measured at baseline, and after 10 and 20 days of consumption. When those in the bottom third of the depressed/elated dimension at baseline were considered, they selectively responded by reporting themselves as happy rather than depressed after taking the probiotic. An unexpected and possibly chance finding was that the consumption of probiotic resulted in a slightly-poorer performance on two measures of memory.

In summary, a theoretical basis for a probiotic treatment trial in major depression is being built up.

\section{Obsessive-compulsive disorder (OCD)}

A hypothesis proposed that stress and antibiotics are the mechanisms by which gut microbiota are altered before the onset of OCD symptoms (Rees 2014). The author proposes a novel explanation of the OCD symptoms that occasionally develop as a part of the PANDAS (Pediatric autoimmune neuropsychiatric disorders associated with streptococcal infections). It is currently believed that these symptoms are explained by group A beta-hemolytic streptococcal infections. However, the author proposes that the symptoms are instead caused by antibiotics that are administered as treatment of the infection, but inadvertently alter microbiota.

Furthermore, stressful life events known to trigger OCD, such as pregnancy, are recast to show the possibility of altering gut microbiota prior to onset of OCD symptoms. Suggested treatment for OCD would be the re-introduction of beneficial bacteria modifying the gut microbiome, thereby ameliorating OCD symptoms (Rees 2014).

\section{Neurodevelopmental disorders}

Animal models demonstrate the effects of microbiota on social behavior. The GF mice showed significant social avoidance indicated by a lack of the normal preference for time spent in a chamber containing a mouse versus the alternative empty chamber (Desbonnet et al. 2014). This was accompanied by reduced preference for novel social situations, where GF mice did not demonstrate the normal increase in time spent investigating a novel over a familiar mouse, which 
resembles social cognition deficits observed in patients with neurodevelopmental disorders. Colonization of the gut reversed the observed social avoidance, but it had no effect on preference for social novelty. This indicates that although the effects of GF rearing on the preference for social novelty are permanently established in the pre-weaning period, the development of social avoidance in GF mice is more amenable to microbialbased interventions (probiotics) in later life. Analogous findings were reported for various social interactions between the mice (Desbonnet et al. 2014). These data clarify the importance of microbiota for the development and execution of normal social behaviors.

A mouse model that displays some features similar to human autism spectrum disorder (ASD) exists, and these mice were treated with the human commensal Bacteroides fragilis (Hsiao et al. 2013). The treatment altered microbial composition, and ameliorated defects in communicative, stereotypic, anxiety-like and sensorimotor behaviors. These findings support a gut-microbiota-brain connection and identify a potential probiotic therapy for ASD and perhaps other human neurodevelopmental disorders (Hsiao et al. 2013).

Taken together, studies by Desbonnet et al. (2014) and Hsiao et al. (2013) offer insights into the pathogenesis of neurodevelopmental disorders marked by alterations of sociability. Such disorders include ASD, schizophrenia spectrum disorders, and perhaps other illnesses and syndromes. It appears that abnormalities in the functioning of the gut-microbiota-brain axis may underlie the sociability problems that are the hallmark of these disorders. If so, probiotic treatment could be a potentially useful treatment of problems such as negative symptoms of schizophrenia, whose resistance to currently available pharmacological interventions is legendary.

A theoretical review points out that the pathogenetic pathway marking the development of schizophrenia involves the intestinal tract (Nemani et al 2015). This pathway may be influenced by the gut microbiota. The review suggests that some schizophrenia patients may benefit from a gluten and casein-free diet, and that antibiotics and probiotics might also have a therapeutic potential.

\section{Acknowledgements: None.}

Conflict of interest: None to declare.

\section{Contribution of individual authors:}

\section{References}

1. Benton D, Williams $C \&$ Brown A: Impact of consuming a milk drink containing a probiotic on mood and cognition. Eur J Clin Nutr 2007; 61:355-61.

2. Desbonnet L, Clarke G, Shanahan F, Dinan TG \& Cryan JF: Microbiota is essential for social development in the mouse. Mol Psychiatry 2014; 19:146-8.

3. Dinan TG \& Cryan JF: Melancholic microbes: a link between gut microbiota and depression? Neurogastroenterol Motil 2013; 25:713-9.

4. Dinan TG, Stanton C \& Cryan JF: Psychobiotics: a novel class of psychotropic. Biol Psychiatry 2013; 74:720-6.

5. Dowlati $Y$, Herrmann $N$, Swardfager $W$, Liu H, Sham L, Reim EK et al.: A meta-analysis of cytokines in major depression. Biol Psychiatry 2010; 67:446-57.

6. Gareau MG, Wine E, Rodrigues DM, Cho JH, Whary MT, Philpott DJ et al.: Bacterial infection causes stress-induced memory dysfunction in mice. Gut 2011; 60:307-17.

7. Hsiao EY, McBride SW, Hsien S, Sharon G, Hyde ER, McCue $T$ et al.: Microbiota modulate behavioral and physiological abnormalities associated with neurodevelopmental disorders. Cell 2013; 155:1451-63.

8. Lyte M: Microbial endocrinology in the microbiome-gutbrain axis: how bacterial production and utilization of neurochemicals influence behavior. PLoS Pathog 2013; 9:e1003726.

9. Maes M, Kenis G, Kubera M., De BM, Steinbusch $H$ \& Bosmans E: The negative immunoregulatory effects of fluoxetine in relation to the CAMP-dependent PKA pathway. Int Immunopharmacol 2005; 5:609-18.

10. Mayer EA, Knight R, Mazmanian SK, Cryan JF \& Tillisch K: Gut microbes and the brain: paradigm shift in neuroscience. J Neurosci 2014; 34:15490-6.

11. Messaoudi M, Lalonde R, Violle $N$, Javelot $H$, Desor $D$, Nejdi A et al.: Assessment of psychotropic-like properties of a probiotic formulation (Lactobacillus helveticus R0052 and Bifidobacterium longum R0175) in rats and human subjects. Br J Nutr 2011; 105:755-64.

12. Naseribafrouei A, Hestad K, Avershina E, Sekelja M, Linlokken A, Wilson $R$ et al.: Correlation between the human fecal microbiota and depression. Neurogastroenterol Motil 2014; 26:1155-62.

13. Nemani K, Hosseini GR, McCormick B \& Fan X: Schizophrenia and the gut-brain axis. Prog Neuropsychopharmacol Biol Psychiatry 2015; 56:155-60.

14. Rees JC: Obsessive-compulsive disorder and gut microbiota dysregulation. Med Hypotheses 2014; 82:163-6.

15. Sampson TR \& Mazmanian SK: Control of brain development, function, and behavior by the microbiome. Cell Host Microbe 2015; 17:565-76.

16. Sudo N, Chida Y, AibaY, Sonoda J, Oyama N, Yu XN et al.: Postnatal microbial colonization programs the hypothalamic-pituitary-adrenal system for stress response in mice. J Physiol 2004; 558:263-75.

\author{
Correspondence: \\ Klara Latalova, MD, PhD \\ Faculty of Medicine and Dentistry, Palacky University Olomouc \\ Department of Psychiatry, Olomouc University Hospital \\ I. P. Pavlova 6, Olomouc 77500, Czech Republic \\ E-mail:klaralat@centrum.cz
}

